\title{
Psicooncología
}

ISSN: 1696-7240

\section{Propuesta de un instrumento para la detección del optimismo estratégi- co en cáncer de mama}

\author{
Sílvia Pastells Pujol ${ }^{1}$; Antoni Font Guiteras ${ }^{2, *}$
}

Recibido: 5 de abril de 2019 / Aceptado 5 de junio de 2019

Resumen: Objetivo: Elaborar un instrumento que nos permita evaluar algunas estrategias cognitivas que formarían parte de las personas optimistas ante una dificultad de salud como el cáncer de mama. Método: 96 pacientes, de ellas 30 se encontraban en una fase de intervalo libre del cáncer de mama, 35 recibiendo tratamiento activo para el cáncer de mama y 31 tenían un diagnóstico de trastorno adaptativo sin historial médico oncológico. Se han comparado los mecanismos cognitivos del instrumento para valorar el optimismo estratégico entre los grupos de salud; se ha efectuado el análisis factorial del instrumento y se ha observado la relación de éste con variables como la calidad de vida y el optimismo disposicional en cada grupo. Resultados: el análisis factorial muestra cuatro componentes del total de ítems del optimismo estratégico: 1) Expectativas de resultado positivo y percepción de control ante la dificultad de salud; 2) Valoración positiva del pasado en el momento actual de salud; 3) Benefit finding a raíz de la enfermedad; y 4 ) Responsabilidad/culpabilidad por la situación de salud. La varianza total explicada por los factores es del 70,67\%. Los factores del optimismo estratégico estarían relacionados con una mejor calidad de vida de las pacientes $(p<0,01 ; r=0,591)$, mostrando relaciones variables en función de la gravedad de la situación médica. Conclusión: el instrumento aportado para evaluar el optimismo estratégico permite identificar estilos cognitivos, relacionados con el mantenimiento de la calidad de vida en cáncer de mama, que pueden ser de utilidad para potenciar las terapias psicológicas de apoyo.

Palabras clave: cáncer de mama, optimismo estratégico, calidad de vida

\section{[en] Proposal for an instrument for the detection of strategic optimism in breast cancer}

\footnotetext{
Abstract: Objective: To elaborate an instrument that allows us to evaluate some cognitive strategies that would be part of the optimistic people facing a health difficulty such as breast cancer. Method: 96 patients, 30 of them were in a breast cancer free interval phase, 35 were receiving active treatment for breast cancer and 31 patients had a diagnosis of adaptive disorder with no oncological medical history. The cognitive mechanisms of the instrument have been compared to evaluate the strategic optimism among the three health conditions. The factorial analysis of the instrument has been carried out and its relationship with variables such as quality of life and dispositional optimism in each group has been

1 Sílvia Pastells Pujol. Departamento de Psicología Básica, Evolutiva y de la Educación - Edificio B. 08193 Bellaterra (Cerdanyola del Vallès). Barcelona $\cdot$ Spain

E-mail: silviapastells@copc.cat

2 Antoni Font. Facultat de Psicologia. Carrer de la Fortuna s/n. Edifici B. Despatx B5b-073 Campus de la UAB. 08193 Bellaterra (Cerdanyola del Vallès). Barcelona. Spain.

E-mail: Antonio.Font@uab.cat

* Dirección de correspondencia: Antoni Font. Facultat de Psicologia. Carrer de la Fortuna s/n. Edifici B. Despatx B5b-073 Campus de la UAB ·08193 Bellaterra (Cerdanyola del Vallès) · Barcelona · Spain.

E-mail: Antonio.Font@uab.cat
} 
observed. Results: the factor analysis shows four components of the total items of strategic optimism: 1) Expectations of positive results and perception of control in front of health difficulties; 2) Positive evaluation of the past in the current health moment; 3 ) Benefit finding in the wake of the disease; and 4) Responsibility / guilt for the current health situation. The total variance explained by the factors is $70.67 \%$. The factors of strategic optimism would be related to a better quality of life of patients ( $p$ $<0.01 ; r=0.591$ ), showing distinct relationships depending on the severity of the medical situation. Conclusion: The provided instrument to assess strategic optimism allows the identification of specific thoughts related to the maintenance of the quality of life in breast cancer, which may be useful in supportive therapies.

Key words: breast cancer, strategic optimism, quality of life

Sumario: 1- Introducción 2. Método 3. Análisis 4. Resultados 6. Discusión 7. Conclusión 8. Referencias bibliográficas

Cómo citar: Pastells Pujol S, Font Guiteras A. Propuesta de un instrumento para la detección del optimismo estratégico en cáncer de mama. Psicooncología 2019; 16: 315-328. doi: 10.5209/psic.65593

\section{Introducción}

El diagnóstico y tratamiento del cáncer de mama conlleva enfrentarse a una serie de acontecimientos estresantes y cambiantes que exigen importantes esfuerzos de adaptación a lo largo del proceso oncológico. Qué aspectos de la vida de las pacientes van a resultar afectadas depende de multitud de factores, observándose importantes diferencias individuales incluso ante situaciones médicas similares ${ }^{(1)}$. El apoyo emocional percibido ${ }^{(2-4)}$, el estado físico previo, las diferentes estrategias de afrontamiento utilizadas por las pacientes ${ }^{(4,5)}$, o características vinculadas a la personalidad como la tendencia al optimismo, son variables que podrían estar relacionadas con la calidad de vida de las pacientes de cáncer de mama ${ }^{(3,5,6)}$.

La influencia del optimismo en la salud de la población general se manifestaría a través de diferentes vías como: una conducta más sana en comparación con los pesimistas, una mayor percepción de apoyo social y una menor percepción de amenaza. De hecho, numerosas investigaciones indican que el rasgo "optimismo" correlaciona incluso con factores biológicos como sufrir menos inflamación y con niveles más saludables de lípidos en sangre ${ }^{(7-9)}$.

Diversos estudios anteriores con pacientes de cáncer de mama relacionan el optimismo disposicional (entendido como rasgo) con una mejor adaptación psicológica . Las pacientes optimistas perciben un mayor control de la situación y presentan estilos de vida más saludables ${ }^{(10,11)}$. En pacientes de cáncer de mama avanzado, el optimismo se asocia a una mejor calidad de vida psicológica ${ }^{(12)}$. Las mujeres con cáncer de mama más optimistas consideran que pasar por la situación de enfermedad les aporta un crecimiento y perciben beneficios de la situación ${ }^{(13-15)}$.

Pese a que numerosos estudios relacionan el optimismo con la calidad de vida, menos atención ha recibido el aspecto estratégico del mismo, es decir, la posibilidad de utilizar el optimismo como estrategia para el mantenimiento de la calidad de vida. Según Avia y Vázquez ${ }^{(16,17)}$, el optimismo (inteligente) se compondría de los siguientes elementos cognitivos: estimación positiva de probabilidades, memoria selectiva, análisis de las causas (atribución positiva), e ilusión de control. Otro 
elemento relevante para la explicación del optimismo sería la búsqueda de un sentido positivo a la enfermedad, como propone Taylor en sus estudios con pacientes de cáncer de mama ${ }^{(18)}$.

Generalmente el optimismo en cáncer de mama se ha evaluado como disposición, con el LOT-R (Revised Life Orientation Test) ${ }^{(19)}$. No obstante, al menos en nuestro entorno, no se dispone de un sistema evaluativo específico para analizar conjuntamente los posibles componentes vinculados a la utilización del optimismo como estrategia, lo que podría ser de gran utilidad para ayudar a las pacientes menos optimistas en su adaptación a la enfermedad.

Por todo ello, el objetivo de este trabajo es desarrollar una propuesta de instrumento para valorar elementos cognitivos que configurarían el optimismo como estrategia ante problemas crónicos de salud como el cáncer de mama.

\section{Método}

\section{Sujetos y procedimiento}

La muestra del presente estudio está formada por 96 pacientes. Las pacientes con un diagnóstico de cáncer de mama $(n=65)$ representan el $67,7 \%$, fueron tratadas mayoritariamente en el Hospital Duran y Reynals y contactaron con la investigadora principal a través de la Asociación Española contra el Cáncer en Barcelona, o fueron derivadas a través de centros de atención primaria al servicio de Psicología en Barcelona en el que trabaja la investigadora, y del que procede también el grupo de pacientes con problemas de adaptación $(n=31)$. Tras el primer contacto, las pacientes eran informadas del estudio y en caso de querer participar, pasaban a ser entrevistadas con previo consentimiento firmado.

Las pacientes de la muestra cumplen los siguientes criterios: 1) Tener un diagnóstico de cáncer de mama y estar en el momento de la entrevista en una de estas dos situaciones: recibiendo tratamiento activo para el cáncer de mama, o encontrarse en fase libre de la enfermedad desde al menos 1 año. O bien, estar en un momento de dificultad personal por estrés adaptativo (según criterios del DSM5), a raíz de situaciones como: separación matrimonial, estrés laboral, dificultades en las relaciones, etc., pero sin haber sufrido ningún cáncer ni tener ningún diagnóstico grave de salud. 2) No sufrir de ninguna enfermedad psiquiátrica que perjudique la comprensión de los procedimientos e instrumentos psicológicos. 3) Ser capaz de poder leer y entender las instrucciones de los cuestionarios.

La edad media de la muestra total fue de 49,40 años (DT: 9,40), el 60,4\% estaba casada o vivía en pareja en el momento de la entrevista, el 71,9\% tenía hijos y el 49 $\%$ estudios básicos (ver Tabla 1).

Los tres grupos de pacientes no muestran diferencias significativas en cuanto a la edad $\left(\chi^{2}=1,045 ; p<0,593\right)$, al nivel de estudios $\left(\chi^{2}=7,439 ; p<0,114\right)$ y tener pareja $\left(\chi^{2}=2,344 ; p<0,310\right)$; pero sí en cuanto a tener hijos (menor número de hijos en el grupo con trastorno adaptativo $\left(\chi^{2}=18,258 ; p<0,001\right)$. Por todo ello, se considera que los tres grupos de pacientes son comparables en cuanto a las características de situación socio demográfica. 
Tabla 1. Características demográficas y médicas de la muestra $(\mathrm{N}=96)$

\begin{tabular}{lll}
\hline Datos & $\boldsymbol{n}$ & $\mathbf{\%}$ \\
\hline & & \\
Pareja & 38 & 39,60 \\
No & 58 & 60,40 \\
Sí & & \\
Hijos & 27 & 28,10 \\
No & 69 & 71,90 \\
Sí & & \\
Nivel de estudios & 47 & 49,00 \\
Básico & 22 & 22,90 \\
Medio & 27 & 28,10 \\
Alto & & 36,46 \\
Situación médica & 35 & 31,25 \\
En tratamiento activo del cáncer de mama & 30 & 32,29 \\
Fase de intervalo libre de cáncer & 31 & \\
Trastorno adaptativo & & \\
\hline
\end{tabular}

\section{Instrumentos}

Valoración del Optimismo Estratégico. Los elementos considerados para la propuesta de ítems del presente instrumento se basan en el trabajo de Avia y Vázquez sobre optimismo inteligente ${ }^{(16,17)}$ y en la teoría de la adaptación cognitiva de Taylor ${ }^{(18)}$.

1) Estimación positiva de probabilidades. La persona optimista tendería a tener expectativas de resultado positivas, independientemente de sus probabilidades objetivas. 2) Ilusión de control. Tendencia a desarrollar creencias de control sobre la situación. 3) Atribución de causalidad. Las personas optimistas se atribuirían a sí mismas las causas de las cosas positivas que les pasan, mientras que no se considerarían la causa de los resultados negativos; los individuos más pesimistas desarrollarían la atribución contraria. 4) Memoria selectiva. Las personas más optimistas tenderían a recordar y recuperar más aspectos positivos de su pasado. 5) Búsqueda de sentido positivo a la experiencia negativa. Las personas buscarían un sentido positivo a la experiencia como estrategia para disminuir los efectos estresantes inmediatos de la misma.

A fin de disponer de un instrumento manejable, cada estrategia se valora mediante tres ítems. En cada ítem la frecuencia de pensamientos se indica mediante valores de 1 (no lo pienso nunca) a 4 (lo pienso continuamente). Todos los ítems hacen referencia al momento actual y al estado de salud. Para valorar la comprensión de los ítems propuestos se realizó en primer lugar una prueba piloto del redactado de los ítems, con 30 pacientes, en la que se constató el adecuado entendimiento del cuestionario. La formulación de los ítems para cada elemento del optimismo estratégico se concretó de la manera siguiente:

1) Expectativas de solución positiva ante el problema de salud: "Me voy a recuperar", "Al final todo esto saldrá bien” y “¡Voy a salir de ésta!”. 
2) Percepción de control ante el problema de salud: "Pienso en lo que puedo hacer para estar mejor", "Puedo superar esta situación de crisis" y "La evolución de la situación depende, en parte, de mí".

3) Responsabilidad/culpabilidad ante el problema de salud: "Soy responsable de la situación", "Esto me ha pasado porque tenía que pasar, por mala suerte.." y "En parte soy la culpable de mi situación".

4) Recuerdos y valoración favorable del pasado: "Me han pasado muchas cosas buenas en la vida", "En mi vida recuerdo más cosas positivas que negativas" y "Casi todo en la vida me ha ido bien".

5) Búsqueda de aspectos positivos a raíz de la situación de salud: "Gracias a la situación valoro aún más el amor de mi gente o el afecto de los demás", "A raíz de la situación, disfruto más de la vida" y "Debido a estas experiencias valoro más las pequeñas cosas de la vida".

Valoración del Optimismo Disposicional. Se ha aplicado el Life Orientation Test- Revised (LOT-R) adaptación española de Otero, Luengo, Romero, Gómez y Castro ${ }^{(19,20)}$. Este cuestionario se compone de 9 ítems ( 3 afirmaciones positivas, 3 negativas y 3 no evaluables) y permite obtener una puntuación directa sobre el denominado optimismo disposicional o predisposición generalizada hacia las expectativas de resultado positivo (a mayor puntuación más optimismo disposicional). Los valores Alfa de Cronbrach obtenidos en el presente trabajo indican que todos los ítems tienen un buen peso acorde con el factor, con valores absolutos entre 0,804 y 0,839. La medida de adecuación muestral de Kaiser-Meyer-Okin (KMO) muestra una puntuación de $0,826(p<0,000)$ y la matriz factorial indica la presencia de un solo factor.

La valoración de la Calidad de vida se ha efectuado con el cuestionario QLCA-Afex de Font y Bayés ${ }^{(21,22)}$, desarrollado y validado de manera satisfactoria en nuestro país. Consta de 27 escalas lineales para evaluar la disminución subjetiva de la calidad de vida en 4 niveles: Síntomas, Autonomía, Familiar/Social y Psicológica/ Emocional, además de una escala de valoración global. En nuestro estudio, el análisis de fiabilidad del instrumento mostró un valor $\alpha$ de Cronbach global de 0,878 . Por escalas se obtuvieron los siguientes valores $\alpha$ de Cronbach: Subescala de Síntomas: 0,856; Subescala de Autonomía (Dificultades con los hábitos cotidianos): 0,869; Subescala de las Dificultades Familiares y Sociales: 0,822; Subescala del Malestar Emocional: 0,828 y Percepción general de afectación de la calidad de vida: 0,884. Así pues, los datos obtenidos sobre la afectación de la calidad de vida presentan un grado adecuado de fiabilidad.

\section{Análisis estadístico}

Los datos fueron analizados mediante el programa estadístico SPSS 15. Para evaluar la validez convergente y discriminante entre los diferentes mecanismos del instrumento se aplicó un análisis de componentes principales (ACP). Para extraer los componentes se eligió la rotación ortogonal o Varimax. La adecuación de la muestra fue evaluada con los coeficientes de Kaiser-Meyer-Olkin (KMO) y de la prueba de esfericidad de Barlett. Para identificar la distribución de los ítems propuestos 
se efectuó un análisis factorial exploratorio (AFE). Para la validez concurrente se analizaron las correlaciones del instrumento para valorar el optimismo estratégico con el optimismo disposicional (LOT-R) y las escalas de la calidad de vida (QLCA-Afex). En este sentido se espera que: a) las correlaciones sean positivas entre el optimismo estratégico y el optimismo disposcional; y b) que las correlaciones sean negativas entre el optimismo estratégico y las escalas de calidad de vida.

\section{Resultados}

El instrumento Optimismo Estratégico presentó un valor global de fiabilidad $\alpha$ de Cronbach de 0,867 . Todos los ítems contribuyen significativamente a la consistencia interna del cuestionario, mostrando valores superiores a 0,7 (ver Tabla 2). La medida de adecuación muestral de Kaiser-Meyer-Olkin (KMO) muestra una puntuación de 0,845 y la prueba de esfericidad de Barlett es significativa $(p<0,001)$. En cuanto a las comunalidades de los ítems, los valores se hallan entre 0,611 y 0,908 lo que indica que la totalidad de los ítems se encuentra relacionado con los factores hallados.

Tabla 2. Coeficientes $\alpha$ de Cronbach del instrumento Optimismo Estratégico

\begin{tabular}{|c|c|c|c|c|c|}
\hline & $\begin{array}{l}\text { Media de la } \\
\text { escala si se } \\
\text { elimina el } \\
\text { elemento }\end{array}$ & $\begin{array}{c}\text { Varianza de } \\
\text { la escala si } \\
\text { se elimina el } \\
\text { elemento }\end{array}$ & $\begin{array}{l}\text { Correlación } \\
\text { elemento-to- } \\
\text { tal corregida }\end{array}$ & $\begin{array}{l}\text { Corre- } \\
\text { lación } \\
\text { múltiple al } \\
\text { cuadrado }\end{array}$ & $\begin{array}{c}\text { Alfa de } \\
\text { Cronbach si } \\
\text { se elimina el } \\
\text { elemento }\end{array}$ \\
\hline $\begin{array}{r}\text { Me han pasado muchas cosas buenas } \\
\text { en la vida }\end{array}$ & 41,60 & 49,617 & 0,645 & 0,755 & $\mathbf{0 , 8 5 2}$ \\
\hline Me voy a recuperar & 41,20 & 49,561 & 0,693 & 0,580 & $\mathbf{0 , 8 5 0}$ \\
\hline $\begin{array}{r}\text { Pienso en lo que puedo hacer para } \\
\text { estar mejor }\end{array}$ & 41,19 & 52,445 & 0,540 & 0,423 & 0,858 \\
\hline Soy responsable de la situación & 41,42 & 55,692 & 0,105 & 0,545 & $\mathbf{0 , 8 8 3}$ \\
\hline $\begin{array}{r}\text { Gracias a la situación valoro aún más } \\
\text { el amor de mi gente o el afecto de } \\
\text { los demás }\end{array}$ & 41,32 & 51,305 & 0,481 & 0,447 & 0,860 \\
\hline $\begin{array}{r}\text { En mi vida recuerdo más cosas posi- } \\
\text { tivas que negativas }\end{array}$ & 41,51 & 49,144 & 0,651 & 0,739 & 0,851 \\
\hline Al final todo esto saldrá bien & 41,29 & 49,387 & 0,768 & 0,732 & 0,847 \\
\hline Puedo superar esta situación de crisis & 41,29 & 50,761 & 0,700 & 0,676 & 0,851 \\
\hline $\begin{array}{r}\text { Esto me ha pasado porque tenía que } \\
\text { pasar, por mala suerte }\end{array}$ & 41,54 & 55,143 & 0,224 & 0,183 & 0,872 \\
\hline Voy a salir de ésta & 41,24 & 50,545 & 0,676 & 0,733 & 0,851 \\
\hline $\begin{array}{l}\text { En parte soy la culpable de mi si- } \\
\text { tuación }\end{array}$ & 41,23 & 54,828 & 0,202 & 0,480 & 0,875 \\
\hline Casi todo en la vida me ha ido bien & 41,61 & 49,904 & 0,618 & 0,658 & $\mathbf{0 , 8 5 3}$ \\
\hline $\begin{array}{r}\text { A raíz de la situación, disfruto más } \\
\text { de la vida }\end{array}$ & 41,63 & 49,103 & 0,659 & 0,576 & $\mathbf{0 , 8 5 1}$ \\
\hline $\begin{array}{l}\text { La evolución de la situación depen- } \\
\text { de, en parte, de mi }\end{array}$ & 41,37 & 52,332 & 0,442 & 0,493 & 0,862 \\
\hline $\begin{array}{l}\text { Debido a estas experiencias valoro } \\
\text { más las pequeñas cosas de la vida }\end{array}$ & 41,25 & 52,407 & 0,515 & 0,561 & 0,859 \\
\hline
\end{tabular}


El análisis de matriz de componentes realizado mediante rotación Varimax obtuvo una solución de 4 factores (ver Tabla 3), con un porcentaje de varianza acumulada del $70,67 \%$. El resultado de este análisis indica que los ítems se distribuyen en los siguientes factores:

Factor 1. Formado por los ítems 2 ("Me voy a recuperar"), 3 ("Pienso en lo que puedo hacer para estar mejor"), 7 (“Al final todo ésto saldrá bien"), 8 ("Puedo superar esta crisis de salud"), 10 ("Voy a salir de ésta) y 14 ("La evolución de la situación de salud depende, en parte, de mí"). Atendiendo a los ítems que lo componen, denominamos a este factor: "Expectativas positivas de resultado y percepción de control".

Factor 2. Formado por los ítems 1 ("Me han pasado muchas cosas buenas"), 6 ("En mi vida recuerdo más cosas positivas") y 12 ("Casi todo me ha ido bien en la vida"). Denominamos "Recuerdos positivos" a este factor.

Factor 3. Formado por los ítems 5 ("A raíz de la enfermedad valoro más el amor de mi gente"), 13 ("Disfruto más de la vida") y 15 ("Valoro más las pequeñas cosas de la vida"). A este factor lo hemos denominado "Identificación de aspectos positivos de la situación o benefit finding".

Factor 4. Formado por los ítems 4 ("Soy la responsable de la situación"), 9 ("Ésto ha pasado porque tenía que pasar") y 11 ("En parte, soy culpable de la situación"), que constituirían el factor denominado "Atribución de responsabilidad ante la situación".

Tabla 3. Matriz de los componentes rotados del Optimismo Estratégico

\section{Ítems del Optimismo estratégico}

Componente

Me han pasado muchas cosas buenas en la vida

Me voy a recuperar

Pienso en lo que puedo hacer para estar mejor

Soy responsable de la situación

Gracias a la situación valoro aún más el amor de mi gente o el afecto de los demás

En mi vida recuerdo más cosas positivas que negativas

Al final todo esto saldrá bien

Puedo superar esta situación de crisis

Esto me ha pasado porque tenía que pasar, por mala

suerte

Voy a salir de ésta

En parte soy la culpable de mi situación

Casi todo en la vida me ha ido bien

A raíz de la situación, disfruto más de la vida

La evolución de la situación depende, en parte, de mi

Debido a estas experiencias valoro más las pequeñas cosas de la vida

\begin{tabular}{|c|c|r|r}
\multicolumn{4}{c}{ Componente } \\
\hline 1 & 2 & 3 & \multicolumn{1}{c}{4} \\
\hline 0,259 & 0,859 & 0,218 & $-0,019$ \\
\hline 0,724 & 0,242 & 0,223 & 0,162 \\
0,710 & 0,231 & 0,026 & $-0,098$ \\
\hline 0,048 & $-0,062$ & 0,005 & 0,892 \\
\hline 0,090 & 0,267 & 0,773 & 0,094 \\
\hline 0,329 & 0,798 & 0,260 & $-0,118$ \\
\hline 0,734 & 0,353 & 0,281 & 0,093 \\
\hline 0,760 & 0,227 & 0,205 & 0,181 \\
\hline 0,374 & $-0,141$ & 0,022 & 0,383 \\
\hline 0,818 & 0,242 & 0,136 & 0,028 \\
\hline 0,040 & 0,086 & 0,067 & 0,851 \\
\hline 0,292 & 0,847 & 0,128 & 0,006 \\
\hline 0,484 & 0,168 & 0,665 & $-0,024$ \\
\hline 0,530 & 0,193 & 0,309 & $-0,434$ \\
\hline 0,168 & 0,114 & 0,890 & $-0,020$ \\
\hline
\end{tabular}

Método de extracción: Análisis de componentes principales. Método de rotación: Normalización Varimax con Kaiser 
Los índices de fiabilidad de los factores identificados obtuvieron los siguientes valores alfa de Cronbach: Expectativas positivas de resultado y Percepción de control ante la situación de salud ( $\alpha=0,875)$, Recuerdos positivos $(\alpha=0,905)$, Identificación de aspectos positivos de la situación o benefit finding $(\alpha=0,778)$, y Atribución de responsabilidad ante la situación $(\alpha=0,664)$.

Se han efectuado análisis de correlación entre el optimismo estratégico y aspectos como la calidad de vida y el optimismo disposicional de las pacientes de la muestra, para comprobar si el instrumento objeto de esta investigación muestra diferencias ante las distintas situaciones médicas.

La relación entre los diferentes mecanismos del optimismo estratégico y el optimismo disposicional muestra valores significativos $(p<0,01)$ (Tabla 4). Las pacientes más optimistas disposicionalmente valoran más frecuentemente su pasado de forma positiva (valores entre $r=0,490$ y $r=0,574$ ), presentan más expectativas de resultado positivo y perciben mayor control ante la situación de salud (valores entre $r=0,424$ y $r=0,685$ ), en los tres grupos. En cambio, una relación significativa entre el optimismo disposicional y el benefit finding únicamente se observa entre las pacientes que se encuentran bajo tratamiento activo en el momento de la entrevista $(r=0,556 ; p<0,01)$. En cuanto a la estrategia "responsabilidad ante la situación médica" no se observan relaciones significativas en ninguno de los grupos ni en el total de la muestra.

Tabla 4. Correlaciones (Spearman) entre los mecanismos del Optimismo Estratégico y Optimismo Disposicional (LOT-R) en las diferentes situaciones de salud

\begin{tabular}{lcccc}
\hline MECAMISMOS & LOT-R & LOT-R & LOT-R & LOT-R \\
$\begin{array}{l}\text { OPTIMISMO } \\
\text { ESTRATÉGICO }\end{array}$ & $\begin{array}{c}\text { MUESTRA } \\
\text { TOTAL }\end{array}$ & $\begin{array}{c}\text { TRATAMIENTO } \\
\text { ACTIVO }\end{array}$ & $\begin{array}{c}\text { INTERVALO } \\
\text { LIBRE }\end{array}$ & $\begin{array}{c}\text { TRASTORNO } \\
\text { ADAPTATIVO }\end{array}$ \\
& $(\mathrm{n}=96)$ & $(\mathrm{n}=35)$ & $(\mathrm{n}=30)$ & $(\mathrm{n}=31)$ \\
\hline $\begin{array}{l}\text { RECUERDOS } \\
\text { BENEFIT FINDING }\end{array}$ & $\mathbf{0 , 4 7 1 * *}$ & $\mathbf{0 , 5 7 4 * *}$ & $\mathbf{0 , 5 2 8 * *}$ & $\mathbf{0 , 4 9 0 * *}$ \\
$\begin{array}{l}\text { EXPECTATIVAS } \\
\text { POSITIVAS Y DE }\end{array}$ & $\mathbf{0 , 2 3 6 *}$ & $\mathbf{0 , 5 5 6 * *}$ & 0,255 & 0,031 \\
CONTROL & $\mathbf{0 , 5 2 1 * *}$ & $\mathbf{0 , 5 8 0 * *}$ & $\mathbf{0 , 6 8 5 * *}$ & $\mathbf{0 , 4 2 4 *}$ \\
& & & & \\
RESPONSABILIDAD & 0,190 & 0,123 & $-0,029$ & 0,134 \\
& & & & \\
\hline
\end{tabular}

* La correlación es significativa al nivel 0,$05 ; * *$ La correlación es significativa al nivel 0,01

En las tablas 5, 6 y 7 se indican las relaciones entre los componentes del optimismo estratégico y la calidad de vida relacionada con la salud en cada grupo de pacientes. Las relaciones son negativas, es decir, a mayor frecuencia de utilización de la estrategia, menor pérdida de calidad de vida. Sin embargo, se observan algunas diferencias en función del grupo de salud. 
En el grupo de pacientes con tratamiento activo del cáncer de mama se observan correlaciones significativas entre las escalas de calidad de vida y las del optimismo estratégico, excepto con la escala de dificultades emocionales. La estrategia "responsabilidad de la situación" no correlaciona significativamente con ninguna de las escalas de calidad de vida (Tabla 5).

Tabla 5. Calidad de vida y mecanismos del Optimismo estratégico. Pacientes en tratamiento activo del cáncer de mama ( $\mathrm{n}=35)$ (Coeficiente de correlación de Spearman).

\begin{tabular}{lcccc}
\hline $\begin{array}{l}\text { ESCALAS } \\
\text { CALIDAD DE } \\
\text { VIDA }\end{array}$ & $\begin{array}{c}\text { RECUERDOS } \\
\text { POSITIVOS }\end{array}$ & $\begin{array}{c}\text { EXPECTATIVAS } \\
\text { POSITIVAS Y } \\
\text { DE CONTROL }\end{array}$ & $\begin{array}{c}\text { BENEFIT } \\
\text { FINDINGS }\end{array}$ & $\begin{array}{c}\text { RESPONSABILIDAD } \\
\text { DE LA SITUACIÓN }\end{array}$ \\
\hline SÍNTOMAS & $-0,172$ & $\mathbf{- 0 , 5 0 4 *}$ & $\mathbf{- 0 , 4 0 4 *}$ & $-0,352$ \\
AUTONOMIA & $\mathbf{- 0 , 4 5 5 *}$ & $\mathbf{- 0 , 3 6 7 *}$ & $\mathbf{- 0 , 4 0 6 *}$ & 0,079 \\
SOCIAL & $\mathbf{- 0 , 4 4 1 ^ { * }}$ & $\mathbf{- 0 , 5 3 7 *}$ & $\mathbf{- 0 , 5 2 6 *}$ & $-0,016$ \\
EMOCIONAL & $-0,296$ & $-0,328$ & $-0,371$ & $-0,176$ \\
GLOBAL & $-0,274$ & $\mathbf{- 0 , 4 5 3 *}$ & $\mathbf{- 0 , 5 3 2 *}$ & $-0,039$ \\
\hline
\end{tabular}

* La correlación es significativa al nivel 0,$05 ; * *$ La correlación es significativa al nivel 0,01

Las relaciones entre la calidad de vida de las pacientes que se hallan en un intervalo libre del cáncer de mama y los factores o mecanismos identificados de optimismo estratégico se indican en la tabla 6. En esta situación médica, valorar el pasado de forma más positiva, esperar con más frecuencia resultados positivos, y percibir más control y más aspectos positivos en la situación, correlaciona significativamente con menor pérdida de calidad de vida, mientras que la pérdida de autonomía no se relaciona significativamente con ninguno de los componentes del optimismo estratégico.

Tabla 6. Calidad de vida y mecanismos del Optimismo Estratégico. Pacientes en Intervalo libre del cáncer de mama ( $\mathrm{n}=30)$ (Coeficiente de correlación de Spearman)

\begin{tabular}{lcccc}
\hline $\begin{array}{l}\text { ESCALAS } \\
\text { CALIDAD DE } \\
\text { VIDA }\end{array}$ & $\begin{array}{c}\text { RECUERDOS } \\
\text { POSITIVOS }\end{array}$ & $\begin{array}{c}\text { EXPECTATIVAS } \\
\text { POSITIVAS Y DE } \\
\text { CONTROL }\end{array}$ & $\begin{array}{c}\text { BENEFIT } \\
\text { FINDINGS }\end{array}$ & $\begin{array}{c}\text { RESPONSABILIDAD } \\
\text { DE LA SITUACIÓN }\end{array}$ \\
\hline SÍNTOMAS & $\mathbf{- 0 , 4 5 1 ^ { * }}$ & $\mathbf{- 0 , 4 3 1 ^ { * }}$ & $\mathbf{- 0 , 4 0 8 *}$ & 0,204 \\
AUTONOMIA & $-0,217$ & $-0,340$ & $-0,315$ & 0,264 \\
SOCIAL & $\mathbf{- 0 , 4 6 2 *}$ & $\mathbf{- 0 , 4 5 6 *}$ & $\mathbf{- 0 , 6 0 7 *}$ & $-0,013$ \\
EMOCIONAL & $\mathbf{- 0 , 6 1 2 * *}$ & $\mathbf{- 0 , 4 4 2 *}$ & $\mathbf{- 0 , 4 7 1 ^ { * }}$ & 0,027 \\
GLOBAL & $\mathbf{- 0 , 5 4 7 ^ { * }}$ & $-0,280$ & $\mathbf{- 0 , 3 7 6 *}$ & 0,041 \\
\hline
\end{tabular}

* La correlación es significativa al nivel 0,$05 ; * *$ La correlación es significativa al nivel 0,01

En cuanto a las pacientes con trastorno adaptativo (Tabla 7), se observan relaciones con un grado mínimo de significación $(p<0,05)$. 
Tabla 7. Calidad de vida y mecanismos del Optimismo Estratégico. Pacientes con Trastorno adaptativo ( $\mathrm{n}=31)$ (Coeficiente de correlación de Spearman)

\begin{tabular}{lcccc}
\hline $\begin{array}{l}\text { ESCALAS } \\
\text { CALIDAD DE } \\
\text { VIDA }\end{array}$ & $\begin{array}{c}\text { RECUERDOS } \\
\text { POSITIVOS }\end{array}$ & $\begin{array}{c}\text { EXPECTATIVAS } \\
\text { POSITIVAS Y DE } \\
\text { CONTROL }\end{array}$ & $\begin{array}{c}\text { BENEFIT } \\
\text { FINDINGS }\end{array}$ & $\begin{array}{c}\text { RESPONSABILIDAD } \\
\text { DE LA SITUACIÓN }\end{array}$ \\
\hline SÍNTOMAS & $\mathbf{- 0 , 5 1 7 ^ { * }}$ & $\mathbf{- 0 , 4 7 9 *}$ & $\mathbf{- 0 , 5 4 0 *}$ & $-0,184$ \\
AUTONOMIA & $-0,309$ & $\mathbf{- 0 , 3 7 9 *}$ & $-0,326$ & 0,222 \\
SOCIAL & $\mathbf{- 0 , 4 1 6 *}$ & $\mathbf{- 0 , 4 4 3 *}$ & $\mathbf{- 0 , 4 2 3 *}$ & 0,012 \\
EMOCIONAL & $\mathbf{- 0 , 4 8 9 *}$ & $\mathbf{- 0 , 4 4 7 *}$ & $\mathbf{- 0 , 3 9 2 *}$ & $-0,197$ \\
GLOBAL & $-0,268$ & $-0,299$ & $\mathbf{- 0 , 4 8 8 ^ { * }}$ & 0,034 \\
\hline
\end{tabular}

* La correlación es significativa al nivel 0,$05 ; * *$ La correlación es significativa al nivel 0,01

Por otra parte, como se observa en las tres tablas (5, 6 y 7$)$, el mecanismo o estrategia de atribución de "responsabilidad de la situación médica" no muestra relaciones significativas con ninguna de las escalas de calidad de vida y en ninguna de las tres situaciones médicas.

\section{Discusión}

En el presente trabajo se propone un nuevo instrumento para valorar elementos cognitivos que conforman el optimismo estratégico ante problemas crónicos de salud como el cáncer de mama, conjuntando la posición teórica sobre el optimismo propuesta por Avia y Vázquez, y el trabajo de Taylor con pacientes de cáncer de mama ${ }^{(16-18)}$. Se han incluido únicamente tres ítems para la valoración de cada aspecto, a fin de disponer de una herramienta de valoración fácil de entender y que pueda aplicarse en un tiempo reducido, como requieren las valoraciones sobre aspectos mediadores o moduladores de la calidad de vida en los entornos de salud. Se trata de poder identificar el grado de utilización de estrategias optimistas ante el problema de salud, para fomentar estas respuestas cognitivas en las intervenciones psicológicas de apoyo.

La formulación de los ítems y la comprensión observada (todas las pacientes pudieron contestar a los ítems sin manifestar ninguna duda sobre los mismos), indica que el instrumento propuesto presenta validez aparente y comprensión adecuada.

El análisis factorial del instrumento propuesto para evaluar el optimismo estratégico de las pacientes con cáncer de mama ante la situación médica, muestra los factores más importantes que podrían relacionarse con una forma optimista de afrontar las situaciones difíciles de salud (validez de constructo). Dicho afrontamiento se relaciona con mejor calidad de vida ante la adversidad, a pesar de que la relación entre las estrategias y mecanismos cognitivos y la calidad de vida varía en función del grado de amenaza objetiva en el que se encuentran las pacientes. Por tanto, parecería que el instrumento es capaz de diferenciar un mayor uso de ciertas estrategias en función de la situación médica (validez discriminante). Las correlaciones observadas con el instrumento de optimismo disposicional (LOT-R) aportan validez concurrente. Los valores alfa de Cronbach obtenidos permiten considerar que el grado de fiabilidad y la consistencia interna de la escala son adecuados. 
Los resultados obtenidos mediante los instrumentos utilizados son coherentes con otras investigaciones. Por ejemplo, los niveles de optimismo disposicional detectados en estas pacientes ${ }^{(23,24)}$ o los niveles de afectación de la calidad de vida observada en las pacientes con cáncer de mama ${ }^{(25,26,27)}$. También los valores de optimismo disposicional son parecidos a los observados en otras muestras ${ }^{(28)}$. Asimismo, los resultados de nuestro trabajo indican que el optimismo se relaciona con una mejor calidad de vida, coincidiendo con el estudio de Smith y colaboradores ${ }^{(29)}$. La importancia del componente "benefit finding", especialmente entre el grupo de pacientes con tratamiento activo, coincide con una de las conclusiones de la revisión de Casellas et al. sobre el funcionamiento psicológico positivo en pacientes con cáncer de mama ${ }^{(30)}$. Por otra parte, el componente "atribución de responsabilidad" pese a mostrarse como un factor coherente en la escala de optimismo estratégico, no se relaciona significativamente con ninguna de las subescalas de calidad de vida, lo que indicaría que no parece ser una estrategia adecuada para preservarla: Sentirse más culpable o responsable por la situación no se relaciona con el bienestar en ninguna de las tres situaciones de salud consideradas.

La información obtenida mediante el presente instrumento tiene, a nuestro entender, importantes implicaciones prácticas: Los mecanismos del optimismo estratégico hacen referencia a la forma en que las pacientes valoran su pasado (estrategia recuerdos positivos), presente (Benefit finding o aspectos positivos y de crecimiento percibidos) y futuro (expectativas positivas y de control ante la situación médica). La consideración conjunta de estos tres elementos temporales determinarían un estado de ánimo u otro a la hora de aceptar y afrontar la enfermedad y su proceso.

Una limitación del estudio es el tamaño de la muestra, aunque puede considerarse suficiente para una primera propuesta a modo de estudio piloto, que deberá complementarse en el futuro mediante una muestra más amplia. También consideramos adecuado destacar la validez ecológica de los datos, dado que se refieren a la situación actual en todos los casos y son obtenidos en el momento en que la situación o problema de salud está presente.

\section{Conclusión}

El presente estudio desarrolla una propuesta conceptual en el contexto de la Psicología Positiva aplicada al campo de la Psicooncología, y aporta un instrumento adecuado para la valoración del optimismo como estrategia para hacer frente al cáncer de mama y que se compone de tres factores: valorar el pasado de manera positiva, identificar aspectos beneficiosos en la situación presente y mantener expectativas de control y de resultado positivo ante la situación médica en el futuro.

\section{Referencias bibliográficas}

1. Falo Zamora C, Villar Abelló H, Rodríguez Bruzos E, Mena Cervignon M, Font Guiteras A. Calidad de vida en cáncer de mama metastásico de larga evolución: la opinión de las pacientes. Psicooncología 2014; 2-3: 313-31. doi: 10.5209/rev_PSIC.2014.v11.n23.47391 . 
2. Durá-Ferrandis E, Mandelblatt JS, Clapp J, Luta G, Faul L, Kimmick G et al. Personality, coping and social support as predictors of long-term quality of life trajectories in older breast cancer survivors: CALGB protocol 369901(Alliance). Psychooncology 2017; 26: 1914-21. doi: 10.1002/pon.4404.

3. Applebaum AJ, Stein EM, Lord-Bessen J, Pessin H, Rosenfeld B, Breitbart W. Optimism, social support, and mental health outcomes in patients with advanced cancer. Psychooncology 2014; 23: 299-306. doi: 10.1002/pon.3418.

4. Font A, Cardoso A. Afrontamiento en cáncer de mama: pensamientos, conductas y reacciones emocionales. Psicooncología 2009; 6: 27-42.

5. Brandão T, Schulz MS, Mena Matos P. Psychological adjustment after breast cancer: a systematic review of longitudinal studies. Psychooncology 2017; 26: 917-26. doi: 10.1002/pon.4230.

6. Colby DA, Shifren K. Optimism, mental health, and quality of life: a study among breast cancer patients. Psychol Health Med 2013; 18: 10-20. doi: 10.1080/13548506.2012.686619.

7. Kim ES, Hagan KA, Grodstein F, DeMeo DL, De Vivo I, Kubzansky LD. Optimism and cause-specific mortality: a prospective cohort study. Am J Epidemiol 2017; 185: 21-9. doi: 10.1093/aje/kww182.

8. DuBois CM, Lopez OV, Beale EE, Healy BC, Boehm JK, Huffman JC. Relationships between positive psychological constructs and health outcomes in patients with cardiovascular disease: A systematic review. Int J Cardiol 2015; 265-80. doi: 10.1016/j. ijcard.2015.05.121.

9. Celano CM, Beale EE, Beach SR, Belcher AM, Suarez L, Motiwala SR et al. Associations between psychological constructs and cardiac biomarkers after acute coronary syndrome. Psychosom Med 2017; 79: 318-26. doi: 10.1097/PSY.0000000000000404

10. Finck C, Barradas S, Zenger M, Hinz A. Quality of life in breast cancer patients: Associations with optimism and social support. Int J Clin Health Psychol 2018; 18: $27-$ 34. doi: 10.1016/j.ijchp.2017.11.002.

11. Henselmans I, Sanderman R, Helgeson VS, de Vries J, Smink A, Ranchor AV. Personal control over the cure of breast cancer: adaptiveness, underlying beliefs and correlates. Psychooncology 2010; 19: 525-34. doi: 10.1002/pon.1599.

12. Lam WW, Yeo W, Suen J, Ho WM, Tsang J, Soong I et al. Goal adjustment influence on psychological well-being following advanced breast cancer diagnosis. Psychooncology 2016; 25:58-65. doi: 10.1002/pon.3871.

13. Bozo O, Gündogdu E, Büyükasik-Colak C. The moderating role of different sources of perceived social support on the dispositional optimism-- posttraumatic growth relationship in postoperative breast cancer patients. J Health Psychol 2009; 14: 1009-20. doi: 10.1177/1359105309342295.

14. Kolokotroni P, Anagnostopoulos F, Tsikkinis A. Psychosocial factors related to posttraumatic growth in breast cancer survivors: a review. Women Health 2014; 54: 56992. doi: 10.1080/03630242.2014.899543.

15. McDonough MH, Sabiston CM, Wrosch C. Predicting changes in posttraumatic growth and subjective well-being among breast cancer survivors: the role of social support and stress. Psychooncology 2014; 23: 114-20. doi: 10.1002/pon.3380.

16. Avia MD, Vázquez C. Optimismo inteligente. Madrid: Alianza Editorial; 1998. 384p.

17. Avia MD, Vázquez C. Optimismo inteligente. Madrid: Alianza Editorial; 2011. 400p.

18. Taylor SE. Adjustment to threatening events: A theory of cognitive adaptation. Am Psychol 1983; 38: 1161-73. 
19. Scheier MF, Carver CS, Bridges MW. Distinguishing optimism from neuroticism (and trait anxiety, self-mastery, and self-esteem): a reevaluation of the Life Orientation Test. J Pers Soc Psychol 1994; 67: 1063-78.

20. Otero JM, Luengo A, Romero E, Gómez JA, Castro C. Psicología de la personalidad. Manual de prácticas. Barcelona: Ariel Practicum. 1998. 256p.

21. Font A, Bayés R. Desarrollo de un instrumento para la medida de la calidad de vida en enfermedades crónicas. En: Forns M, Angera MT, coordinadores. Aportaciones recientes a la evaluación psicológica. Barcelona: Promociones y Publicaciones Universitarias; 1993. p.175-195.

22. Font A, Bayés R (dir). Valoración de la calidad de vida en pacientes de cáncer (Tesis doctoral). (Cerdanyola del Vallès, Barcelona): Universitat Autònoma de Barcelonal, 1998.

23. Schou I, Ekeberg Ø, Ruland CM, Sandvik L, Kåresen R. Pessimism as a predictor of emotional morbidity one year following breast cancer surgery. Psychooncology 2004; 13 : 309-20. doi: 10.1002/pon.747.

24. Lam WW, Bonanno GA, Mancini AD, Ho S, Chan M, Hung WK et al. Trajectories of psychological distress among Chinese women diagnosed with breast cancer. Psychooncology 2010; 19: 1044-51. doi: 10.1002/pon.1658.

25. Rodríguez E, Font A. Eficacia de la terapia de grupo en cáncer de mama: evolución de las emociones desadaptativas. Psicooncología 2013; 10: 275- 87. doi: 10.5209/ rev_PSIC.2013.v10.n2-3.43449.

26. Applebaum AJ, Stein EM, Lord-Bessen J, Pessin H, Rosenfeld B, Breitbart W. Optimism, social support, and mental health outcomes in patients with advanced cancer. Psychooncology 2013;23:299-306. doi: 10.1002/pon.3418

27. Colby DA, Shifren K. Optimism, mental health and quality of life: a study among breast cancerpatients. PsycholHealth Med2013;18:10-20. doi: 10.1080/13548506.2012.686619.

28. Hinz A, Sander C, Claesmer H, Brähler E, Zenger M, Hilbert A et al. Optimism and pessimism in the general population: Psychometric properties of the Life Orientation Test (LOT-R). Int J Clin Health Psychol 2017; 17: 161-70. doi: 10.1016/j.ijchp.2017.02.003.

29. Smith S K, Herndon JE, Lyerly HK, Coan A, Wheeler JL, Staley T et al. Brief report: correlates of quality of life-related outcomes in breast cancer patients participating in the Pathfinders pilot study. Psychooncology 2011; 20: 559-64. doi: 10.1002/pon.1770.

30. Casellas-Grau A, Vives J, Font A, Ochoa C. Positive psychological functioning in breast cancer: an integrative review. Breast 2016; 27: 136-68. doi: 10.1016/j.breast.2016.04.001. 
ANEXO 1: Instrumento para la valoración del optimismo estratégico (VOE) En estos momentos de estrés y dificultad de salud creo o pienso que .... (Indica la opción que mejor expresa lo que sientes actualmente)

\begin{tabular}{|c|c|c|c|}
\hline \multicolumn{4}{|c|}{.... me han pasado muchas cosas buenas en la vida } \\
\hline 1. Ninguna vez & 2. Pocas veces & 3. Muchas veces & 4. Continuamente \\
\hline
\end{tabular}

\section{.... me voy a recuperar}
1. Ninguna vez
2. Pocas veces
3. Muchas veces
4. Continuamente

\section{.... pienso en lo que puedo hacer para estar mejor}
1. Ninguna vez
2. Pocas veces
3. Muchas veces
4. Continuamente

\section{.... soy responsable de la situación \\ 1. Ninguna vez 2. Pocas veces}

3. Muchas veces 4. Continuamente

.... gracias a la situación valoro aún más el amor de mi gente o el afecto de los demás
1. Ninguna vez
2. Pocas veces
3. Muchas veces
4. Continuamente

\begin{tabular}{|c|c|c|c|}
\hline \multicolumn{4}{|c|}{.... en mi vida recuerdo más cosas positivas que negativas } \\
\hline 1. Ninguna vez & 2. Pocas veces & 3. Muchas veces & 4. Continuamente \\
\hline
\end{tabular}

\section{.... al final todo esto saldrá bien}
1. Ninguna vez
2. Pocas veces
3. Muchas veces
4. Continuamente

\section{.... puedo superar esta situación de crisis}
1. Ninguna vez
2. Pocas veces
3. Muchas veces
4. Continuamente

\begin{tabular}{|c|c|c|c|}
\hline .... esto me ha & o porque tenía & ar, por mala suer & \\
\hline 1. Ninguna vez & 2. Pocas veces & 3. Muchas veces & 4. Continuamente \\
\hline
\end{tabular}

.... voy a salir de ésta!
1. Ninguna vez
2. Pocas veces
3. Muchas veces
4. Continuamente

\begin{tabular}{|c|c|c|c|}
\hline \multicolumn{4}{|c|}{.... en parte soy la culpable de mi situación } \\
\hline 1. Ninguna vez & 2. Pocas veces & 3. Muchas veces & 4.Continuamente \\
\hline
\end{tabular}

\section{.... casi todo en la vida me ha ido bien}
1. Ninguna vez
2. Pocas veces
3. Muchas veces
4. Continuamente

\section{... a raíz de la situación, disfruto más de la vida}
1. Ninguna vez
2. Pocas veces
3. Muchas veces
4. Continuamente
1... la evolución
1. Ninguna vez
2. Pocas veces
3. Muchas veces
4. Continuamente
... debido a estas experiencias valoro más las pequeñas cosas de la vida
$\begin{array}{lll}\text { 1. Ninguna vez } 2 \text {. Pocas veces } & \text { 3. Muchas veces } \quad \text { 4. Continuamente }\end{array}$

